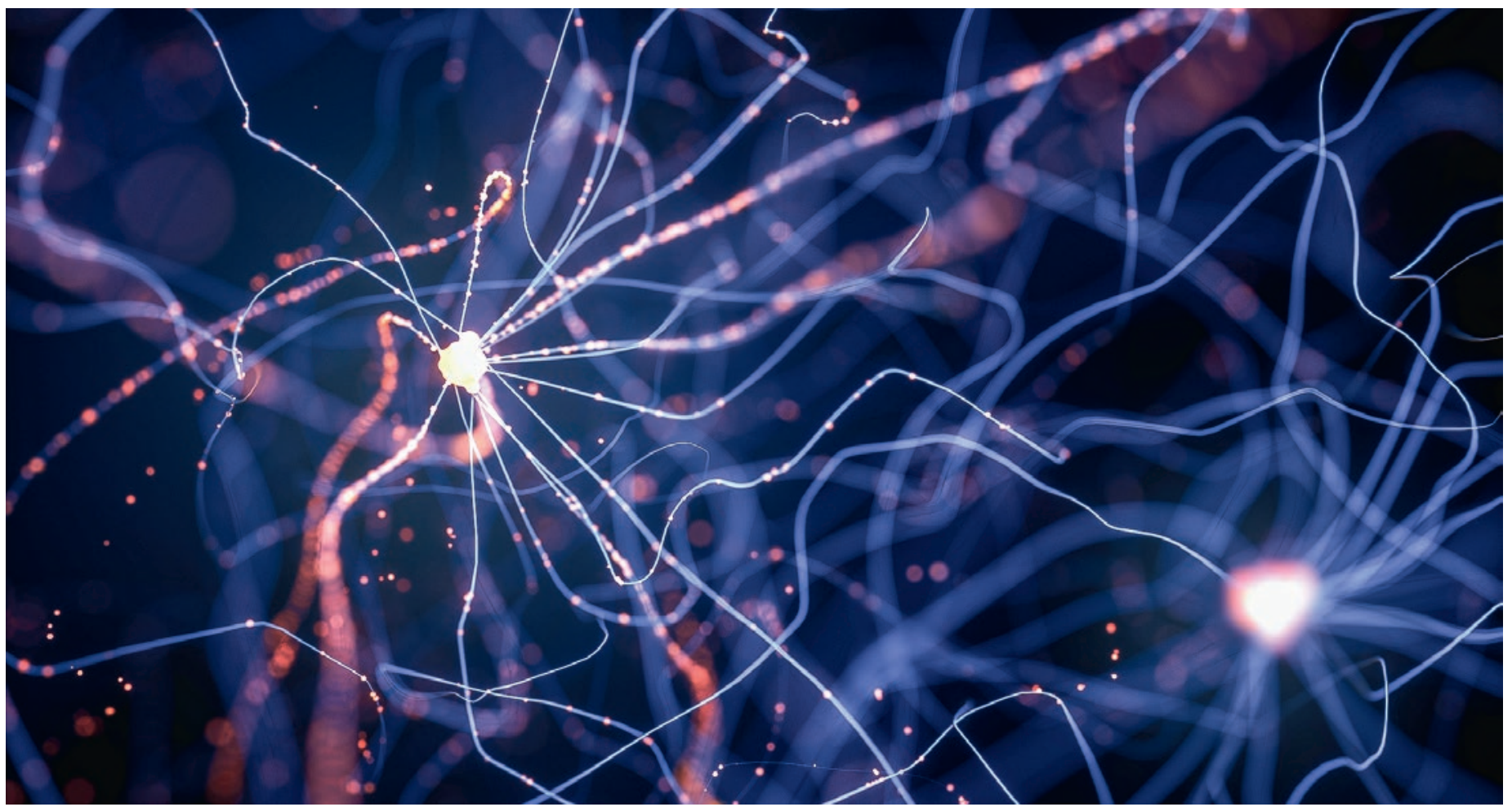

\title{
Expertinnen und Experten zusammenbringen
}

\section{Philippe Lyrer}

Prof. Dr. med., Präsident SFCNS, Universitätsspital Basel

Die Swiss Federation of Clinical Neuro-Societies (SFCNS) vereint 14 Gesellschaften mit mehr als 2000 Expertinnen und Experten der klinischen Neurowissenschaften. Es ist der 2009 gegründeten Föderation ein Anliegen, mit ihren Projekten den Austausch und die Zusammenarbeit zwischen den einzelnen Disziplinen zu fördern, um so dafür zu sorgen, dass Patientinnen und Patienten mit neurologischen Erkrankungen besser behandelt werden.

Nicht nur in den klinischen Neurowissenschaften, in der Medizin im Allgemeinen ist durch medizinischen und technologischen Fortschritt seit einigen Jahren eine Fragmentierung, eine Aufteilung von Disziplinen in Subdisziplinen, zu beobachten, die den Austausch und die Zusammenarbeit zwischen den Fächern zunehmend erschwert. Die SFCNS erachtet es jedoch als wichtig, medizinische Fachpersonen zusammenzubringen und so eine Einheit zwischen den verschiedenen Disziplinen und den ausführenden Spezialistinnen und Spezialisten zu wahren. Die SFCNS fungiert deshalb seit ihrer Gründung vor zwölf Jahren als Plattform für zahlreiche Expertinnen und Experten im Bereich der klinischen Neurowissenschaften.

\section{Gemeinsame Weiterbildung}

Die SFCNS hat sich vor etwas mehr als einem Jahr zum Ziel gesetzt, den Zusammenhalt zu stärken und bei allen Mitgliedsgesellschaften der SFCNS das Bewusstsein dafür zu schärfen, dass sie einer Föderation angehören, die sie unterstützt. Die Föderation ist beispielsweise bei berufspolitischen Fragen behilflich, die vereinte Kräfte erfordern. Aktuell arbeitet die SFCNS etwa an einer gemeinsamen Grundweiterbildung für angehende Fachärztinnen und Fachärzte aus der Neurologie, Neurochirurgie, Neuroradiologie und Neuropädiatrie. Dieser sogenannte Common Trunk hat gegenüber der aktuellen Weiterbildung, welche die vier 
Neurodisziplinen praktisch getrennt voneinander durchführen, einige Vorteile: Mit dem Common Trunk hätten Assistenzärztinnen und -ärzte, die am Anfang ihrer fachärztlichen Weiterbildung stehen, etwa die Möglichkeit, mehrere Neurodisziplinen kennenzulernen, und mehr Zeit, um ihre spätere Fachrichtung zu wählen. Absolvierende würden innerhalb der klinischen Neurodisziplinen eine auf ihre Interessen massgeschneiderte Weiterbildung erhalten.

Gleichzeitig würde eine gemeinsame Weiterbildung aber auch das gegenseitige Verständnis der Fachgebiete verbessern. Neurologinnen und Neurologen könnten zum Beispiel auch spezifische neurochirurgische oder -radiologische Kenntnisse erwerben, was ihnen in der Zusammenarbeit mit Neurochirurginnen oder Neuroradiologen zugutekommt und so natürlich letztlich auch den Patientinnen und Patienten.

Für die Neuroradiologinnen und Neuroradiologen hätte ein Common Trunk den besonderen Vorteil, dass sie sich bereits in einer frühen Phase der Weiterbildung mit dem Nervensystem auseinandersetzen würden. Aktuell handelt es sich bei der Neuroradiologie um ein Schwerpunktfach der Radiologie. Neuroradiologinnen und Neuroradiologen absolvieren heute also

\section{Eine gemeinsame Grundausbildung würde das gegenseitige Verständnis der Fachgebiete verbessern.}

zunächst eine Weiterbildung zur Fachärztin, zum Facharzt für Radiologie und spezialisieren sich erst dann auf die Neuroradiologie. Die gemeinsame Grundausbildung würde die Neuroradiologie auch formal stärker zu einem klinisch-neurowissenschaftlichen Fach machen.

Derzeit kümmern sich die einzelnen Fachgebiete um die Anpassung ihrer jeweiligen Weiterbildungscurricula. Die SFCNS koordiniert diesen Prozess und fördert hierbei ganz im Sinne ihrer Strategie den Dialog zwischen den Disziplinen.

\section{Gemeinsame Veranstaltungen}

Die Föderation wirkt einer Fragmentierung einerseits mit der Entwicklung dieser gemeinsamen Grundausbildung entgegen, andererseits aber auch mit gemeinsamen Veranstaltungen, mit denen der Austausch unter Vertreterinnen und Vertretern derselben, aber auch verschiedener Neurodisziplinen gefördert werden soll. Die wohl bekannteste Veranstaltung der Föderation ist der

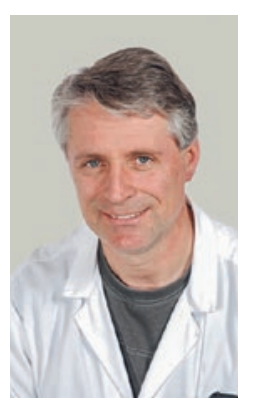

Prof. Dr. med. Philippe Lyrer ist Facharzt für Neurologie und Chefarzt ad interim der Neurologischen Klinik und Poliklinik am Universitätsspital Basel. Der gebürtige Basler ist seit 2019 Präsident der Swiss Federation of Clinical NeuroSocieties (SFCNS), einer 2009 gegründeten Föderation, der 14 Gesellschaften aus dem Neurobereich angehören. Weitere Informationen zur SFCNS erhalten Sie unter www.sfens.ch

SFCNS Congress, der alle drei Jahre stattfindet - das nächste Mal Ende September 2022 in Basel. Die SFCNS organisiert aber auch jährlich einen sogenannten Imaging Course, eine mehrteilige Veranstaltung, die Fachpersonen unterschiedlicher Disziplinen die Bildgebung im Neurobereich näherbringen möchte.

\section{Erfolge nach aussen aufzeigen}

Neben diesen Projekten, welche die Zusammenarbeit innerhalb der klinischen Neurowissenschaften fördern sollen, ist es der SFCNS auch ein Anliegen, sich stärker nach aussen, an die Öffentlichkeit zu richten und die zahlreichen Erfolge der klinischen Neurowissenschaften aufzuzeigen. Denn in den Neurodisziplinen hat sich in den letzten Jahren sehr viel getan, das den Patientinnen und Patienten direkt zugutekommt. Dazu zählt etwa die flächendeckende Versorgung von Hirnschlagpatientinnen und -patienten in hochspezialisierten Stroke Centers und Stroke Units, die es mittlerweile in der ganzen Schweiz gibt. Diese werden unter der Federführung der SFCNS regelmässig zertifiziert und sind ein gutes Beispiel dafür, was die einzelnen Disziplinen der klinischen Neurowissenschaften zugunsten der Schweizer Bevölkerung erreichen können, wenn sie in einer Föderation wie der SFCNS kollaborieren.

\section{Bildnachweis}

Abbildung: Kts | Dreamstime.com

Foto: Universitätsspital Basel

\section{Das Wichtigste in Kürze}

- Die Swiss Federation of Clinical Neuro-Societies (SFCNS) fördert den Austausch und die Zusammenarbeit in den klinischen Neurowissenschaften.

- Aktuelle Projekte sind etwa eine gemeinsame Grundweiterbildung für angehende Fachärztinnen und Fachärzte aus den neurologischen Fachgebieten sowie gemeinsame Veranstaltungen. 